Pol. Con. (Edición núm. 19) Vol. 3 No 5

Mayo 2018, pp. 128-149

ISSN: 2550 - 682X

DOI: $10.23857 / p c . v 3 i 5.491$

Recepción: 22/ 12/ 2017

Aceptación: 19/ 02/ 2018

Publicación: 04/ 05/ 2018

Ciencias Técnicas y Aplicadas

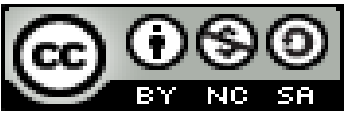

Artículo de Revisión

\title{
Proyecto de diseño de un Plan de Mantenimiento de tanques de almacenamiento: Techo de domo geodésico
}

\section{Design project for a Storage Tank Maintenance Plan: Geodesic dome roof}

\section{Projeto de projeto para um plano de manutenção do tanque de armazenamento: teto geodésico}

\author{
Milton G. Naranjo-Flores ${ }^{\mathrm{I}}$ \\ lobos1989@hotmail.com \\ Lenin S. Orozco-Cantos II \\ 1sorozco@espoch.edu.ec \\ Santiago A. López-Ortiz ${ }^{\text {III }}$ \\ salopez@espoch.edu.ec \\ Milton I. Quinga-Morales ${ }^{\text {IV }}$ \\ miltonquinga@espoch.edu.ec \\ Edwin F. Viteri-Núñez ${ }^{\mathrm{V}}$ \\ eviteri@espoch.edu.ec \\ Gloria E. Miño Cascante VI \\ gmino@espoch.edu.ec
}

Correspondencia:1sorozco@espoch.edu.ec

\footnotetext{
I Ingeniero Mecánico, Escuela Superior Politécnica de Chimborazo, Riobamba, Ecuador.

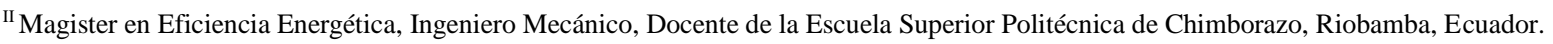

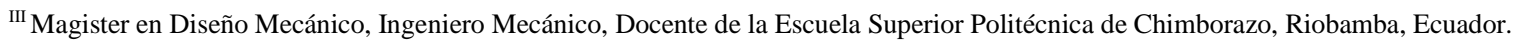

${ }^{\text {IV }}$ Maestro en Ingeniería Automotriz, Ingeniero Automotriz, Docente de la Escuela Superior Politécnica de Chimborazo, Riobamba, Ecuador.

v Magister en Gerencia de Proyectos de Ecoturismo, Ingeniero Mecánico, Docente de la Escuela Superior Politécnica de Chimborazo, Riobamba, Ecuador.

${ }^{\mathrm{VI}}$ Magister en Docencia Universitaria e Investigación Educativa, Magister en Dirección de Empresas Mención Proyectos, Doctor en Ciencias Económicas, Docente de la Escuela Superior Politécnica de Chimborazo, Riobamba, Ecuador.
} 


\section{Resumen}

Este artículo plantea un plan de mantenimiento acorde a las nuevas tecnologías de mantenimiento y que se correspondan con los nuevos tipos de tanques de techo de domo geodésico, que se instalan como parte de la modernización de la planta ubicada en la Refinería Estatal Esmeraldas. Para este proyecto se tomó como referencia la norma API 653, considerando las normas encontradas y analizadas como es RP 579, Std 620, Std 650 RP 651, RP 652, Std 2000, RP 2003, STD 2015, RP 2016, Publ 2201, RP 2207, Publ 2217A, ASME 1, SNT-TC-1A, ASTM3, D1, STD 2610. El análisis de los resultados, permitió obtener un plan de mantenimiento preventivo detallado en actividades, personal, tiempo y frecuencias de realización con costos aproximados a la actualidad de personal, equipos y materiales que se utilizaran en cada una de las actividades de mantenimiento, calculado para toda la vida útil del tanque, para realizar un diagnóstico preventivo para evitar o disminuir daños a largo y corto plazo, en base a sus necesidades y condiciones de operación. Entre sus conclusiones, se propone la necesidad de insertar el plan de mantenimiento de forma integral en la empresa, gestionando la utilización de las tablas de actividades que contienen, frecuencia de tiempo, personal, herramientas, materiales y equipos, el mismo que proporciona la seguridad y calidad para el desarrollo y mejoramiento de sus tanques.

Palabras clave: plan de mantenimiento; instituto americano del petróleo (API 653); planificación del mantenimiento; historial de las inspecciones; tecnologías de mantenimiento.

\section{Abstract}

This article proposes a maintenance plan according to the new maintenance technologies that correspond to the new types of geodesic dome roof tanks, which are installed as part of the modernization of the plant located in the Esmeraldas State Refinery. For this project the API standard 653 was taken as reference, considering the rules found and analyzed as RP 579, Std 620, Std 650 RP 651, RP 652, Std 2000, RP 2003, STD 2015, RP 2016, Publ 2201, RP 2207, Publ 2217A, ASME 1, SNT-TC-1A, ASTM3, D1, STD 2610. The analysis of the results made it possible to obtain a detailed preventive maintenance plan in activities, personnel, time and frequency of execution with approximate costs to the Actual personnel, equipment and materials that will be used in each of the maintenance activities, calculated for the entire life of the tank, to make a preventive diagnosis to avoid or reduce long and short term damage, based on your needs 
and Operating conditions. Among its conclusions, it is proposed the need to insert the maintenance plan holistically in the company, managing the use of tables of activities that contain, frequency of time, personnel, tools, materials and equipment, the same that provides security and quality for the development and improvement of their tanks.

Keywords: maintenance plan; American Petroleum Institute (API 653); maintenance planning; history of inspections; maintenance technologies.

\section{Resumo}

Este artigo propõe um plano de manutenção de acordo com as novas tecnologias de manutenção que correspondem aos novos tipos de tanques de teto geodésico, que são instalados como parte da modernização da planta localizada na Refinaria do Estado de Esmeraldas. Para este projeto foi tomado como referência a API 653 padrão e os regulamentos encontrados e analisados como RP 579, Std 620, Std 650 RP 651, RP 652, Std 2000, a RP 2003 STD 2015, RP 2016, Publ 2201, RP 2207, Publ 2217A, uma ASME SNT-TC-1A, ASTM3, D1, DST 2610. a análise dos resultados obtidos permitiram obter um plano de manutenção preventiva detalhada actividades, de pessoal, tempo e frequência de custos estimados com a forma de realização Pessoal real, equipamentos e materiais que serão utilizados em cada uma das atividades de manutenção, calculados para toda a vida do tanque, para fazer um diagnóstico preventivo para evitar ou reduzir danos a curto e longo prazo, com base em suas necessidades e condições de operação. Entre suas conclusões, propõe-se a necessidade de inserir o plano de manutenção de forma holística na empresa, gerenciando o uso de tabelas de atividades que contenham, frequência de tempo, pessoal, ferramentas, materiais e equipamentos, o mesmo que proporciona segurança. E qualidade para o desenvolvimento e melhoria de seus tanques.

Palavras chave: plano de manutenção; Instituto Americano do Petróleo (API 653); planejamento de manutenção; histórico de inspeções; tecnologias de manutenção.

\section{Introducción}

A nivel mundial en la industria petrolera se utilizan los tanques de almacenamiento, para contener crudo y sus derivados del petróleo ya que son de sumo valor en la explotación de los hidrocarburos y actúan como un pulmón entre la producción y el transporte del petróleo y sus derivados, donde se absorbe las variaciones de consumo ayudando a la refinería a mantener un 
nivel constante entre la producción y el consumo del producto final y una de las operaciones que permite mantener esta constante es mediante un plan de mantenimiento. (Campbell y Abdul, 2000).

Entendido como plan de mantenimiento al conjunto de tareas llevadas a cabo de manera organizada y sistemática, darán como resultado la limpieza o renovación de los equipos de una planta. Se pueden realizar mantenimientos correctivos en el caso de presentar fallas los equipos o componentes operativos de una fábrica o refinería etc. (Torres, 2005). Por otra parte, los mantenimientos preventivos como su nombre lo indica, su función es anteponerse a las fallas o los desgastes para tener los equipos en buenas condiciones operativas sin la necesidad de parar bruscamente las actividades de la misma debido a las fallas de los equipos. (García, 2003). (Batista, 2011).

Para ello, el desarrollo de un plan de mantenimiento para los tanques de almacenamiento de domo geodésico es primordial, ya que en ellos se almacena petróleo que al momento es considerado la principal matriz productiva del Ecuador. Otro aspecto a tomar en cuenta es la seguridad que exigen actualmente los estándares de seguridad laboral y aún más al tratarse de una empresa con una actividad económica tan complicada como la de almacenamiento de crudo para ulteriormente su refinación. (PETROECUADOR, 2000).

En la Refinería Estatal Esmeraldas, ubicada en la provincia de su mismo nombre, han utilizado tanques de almacenamiento de techo fijo y techo flotante a lo largo de sus años de trabajo, por las características propias de los derivados del petróleo y de acuerdo a la producción de la planta de refinación tenemos: el área de almacenamiento y manejo de los hidrocarburos, en las cuales se comenzó a instalar techos de domo geodésicos, que sirve para proteger de las influencias atmosféricas y ambientales mientras que al mismo tiempo, se minimiza la emisión de vapores peligrosos y previniendo la entrada de agua en el tanque.

Por falta de mantenimiento se ha ido produciendo deterioro anticipado, que los techos flotantes tiendan a voltearse; los techos fijos se corroan y formen agujeros, las paredes presentan picaduras, fugas o filtraciones por los accesorios y corrosión externa del fondo de los tanques los mismos causan pérdidas económicas y materiales, debido a esto se producen paros en el proceso para el mantenimiento correctivo donde se detiene la planta para realizar los trabajos necesarios y 
cada día que no produzca la planta provoca pérdidas económicas, debido a estos inconvenientes, lo más perjudicial es la contaminación que se podrían producir al medio ambiente por los probables derrames y gases emitidos que dañan el ecosistema y afecta a la salud de los trabajadores y personas que viven cerca de la Refinería Estatal Esmeraldas. Dado el deterioro, la planta está sustituyendo los tanques y a la fecha se han cambiado cuatro, con ello se ha empleado diferente tecnología de fabricación por lo cual los planes de mantenimiento existentes en la empresa de por si obsoletos, por más de 30 años de elaboración, y sin una actualización muestran la necesidad de elaborar o proponer el plan de mantenimiento a los nuevos tanques. (Batista, 2011).

Con los códigos y normativas internacionales de mantenimiento de tanques de almacenamiento, estos están basados en las experiencias y conocimientos de fabricantes y usuarios de tanques de almacenamiento, soldados de petróleo y sus derivados de distintos tamaños que va relacionada con la capacidad necesaria de la empresa. (Cuatrecasas, 2000). (García, 2003).

\section{Materiales y Métodos}

La capacidad y cantidad de tanques para almacenar los barriles de petróleo en China y Estados Unidos, cubre las existencias récord que hoy registran ambos países en la extracción del producto almacenado. Al momento el bajo costo del crudo no ayuda a la comercialización del producto por lo que los operadores colman los depósitos en espera de un repunte en el precio y hay cada vez más demanda de tanques de almacenamiento de hidrocarburos.

Ecuador con sus tres refinerías, tiene una capacidad total de refinación de 175 mil barriles diarios, siendo la más grande la "Refinería de Esmeraldas" con 110 mil barriles diarios, seguida de "La Libertad" y "Amazonas" con 45 y 20 mil barriles diarios de capacidad respectivamente. (Betún y col, 2010). (Naranjo, 2016).

Ante la necesidad, de garantizar la medición y propiedades de los hidrocarburos, se han modernizado y se encuentran diversos tipos de tanques, que se acoplan a los requerimientos y parámetros establecidos en el momento de almacenar el producto. Ante ello, esta propuesta presenta la posibilidad de aclarar los conceptos de diseño, plan y mantenimiento de tanques de almacenamiento de techo de domo geodésico, sujetos a normas internacionales de 

estandarización API, específicamente las normativas API 653 para el desarrollo de este proyecto hacen referencia a los requisitos que se debe cumplir en las inspecciones, reparaciones, modificaciones y nuevos traslados de los tanques que han sido construidos por la normativa API 650.

API 653 está pensado para condiciones de funcionamiento de los tanques, elimina las trabas que se puede aplicar para las labores de mantenimiento, es un código pensado para la fabricación de cada componente. Es considerada una norma autónoma ya que, en el caso de un aparente conflicto entre la norma API 653 y API 650 prevalece la norma API 653 durante la labor antes descrita. (Mayorga, 2013). (Murray, 1997).

\section{API 653 descripción y alcance}

Abarca tanques de acero que sirven para almacenar y son construidos en base a la norma API 650 y API 12C. Provee ciertos requisitos para mantener su estructura de forma íntegra de los tanques, luego de que hayan entrado en servicio, además abarca la observación, su reconstrucción y/o su reparación. El alcance está limitado a la fundación del tanque, fondo, cuerpo, estructura, techo y aditamentos agregados a la cara de la primera brida, primera unión roscada o soldada. (Dounce, 2000). (ENERGY, 2015).

Muchos de los diseños, soldaduras, inspección y los requerimientos de los materiales de acuerdo con API 650, pueden ser aplicados en la inspección de mantenimiento, toma de datos, reparaciones, y alteraciones de tanques en servicio. En el caso de aparentes conflictos entre los requisitos de este estándar y API 650 o su predecesor API 2.2D, este estándar deberá predominar para tanques que han sido puestos en servicio de esta forma se obtendrá los resultados esperados.

Este estándar no contiene reglas o pautas que cubran todas las condiciones variables, que pueden ocurrir o existir en un tanque. Cuando los detalles del diseño y de construcción no se conocen, y está disponible el estándar como construido, los detalles que proporcionan un nivel de integridad igual al nivel proporcionado por la edición actual de API Std. 650 deben ser utilizados.

\section{API 650 descripción y alcance}

Esta norma cubre los requerimientos para la fabricación, diseño, materiales, montaje y algunas pruebas ejecutadas para tanques verticales cilíndricos soldados, sobre la superficie con extremo 
superior abierto o cerrado en diversos volúmenes y tonelajes con presiones internas similares a la presión atmosférica (no deben exceder el peso de las láminas del techo), pero se permiten presiones internas más altas cuando se cumplen requerimientos adicionales. (ENERGY, 2015).

Aplica para tanques en los cuales la totalidad del fondo del tanque está soportado uniformemente y para tanques en servicio no refrigerado, que tienen una temperatura máxima de diseño de $90^{\circ} \mathrm{C}$ o menos. El API-650, sirve para aplicar técnicas estandarizadas para la fabricación de tanques con normas de seguridad adecuada y costos razonables para el almacenamiento de petróleo y sus derivados y otros productos líquidos comúnmente usados y almacenados por la industria. Este código no establece dimensiones definidas de tanques se puede escoger cualquier tamaño que se necesite.

A continuación, en la Tabla 1, se enlistan varios de los códigos aplicables a tanques de almacenamiento. (ENERGY, 2015).

Tabla 1. Códigos y estándares aplicables en el mantenimiento de tanques de almacenamiento

\begin{tabular}{|l|l|}
\hline \multicolumn{2}{|c|}{ Principales códigos y estándares } \\
\hline Código & Aplicación \\
\hline RP 579 & Apropiado para uso (Fitness-forservices). \\
\hline Std 620 & $\begin{array}{l}\text { Diseño y construcción de tanques de almacenamiento grande, soldado y } \\
\text { de baja presión. }\end{array}$ \\
\hline Std 650 & Tanques soldados de acero para almacenamiento de crudo. \\
\hline RP 651 & Proteccion catodica para tanques de almacenamiento sobre el suelo \\
\hline RP 652 & $\begin{array}{l}\text { Recubrimiento de los fondos de tanques sobre el suelo para } \\
\text { almacenamiento de petroleo }\end{array}$ \\
\hline Std 2000 & $\begin{array}{l}\text { Venteo atmosferico y tanque de almacenamiento de baja presion. No } \\
\text { refrigerados y refrigerados. }\end{array}$ \\
\hline RP 2003 & $\begin{array}{l}\text { Proteccion contra ignicion sugiriendo de estatica, rayos y correntes } \\
\text { extraviadas }\end{array}$ \\
\hline STD 2015 & $\begin{array}{l}\text { Entrada segura y limpieza de tanques de almacenamiento de petroleo } \\
\text { almacenamiento de petroleo }\end{array}$ \\
\hline RP 2016 & \begin{tabular}{l} 
Practica recomentada para el ingreso y limpieza de tanque de \\
\hline
\end{tabular}
\end{tabular}




\begin{tabular}{|c|c|}
\hline Publ 2201 & Procedimiento para soldadura o hot tapping en equipos en servicio \\
\hline RP 2207 & Preparacion de fondos de tanques para trabajos en caliente \\
\hline Publ 2217A & $\begin{array}{l}\text { Lineamientos para el trabajo en espacios confinados en la industria del } \\
\text { petroleo }\end{array}$ \\
\hline ASME 1 & $\begin{array}{l}\text { Codigo de calderas y recipientes a presion. } \\
\text { Seccion V, ensayos no destructivos } \\
\text { Seccion VIII, recipientes a presion } \\
\text { Reglas alternativas division } 2 \\
\text { Seccion IX, calificacion y soldadura fuerte (brazing) }\end{array}$ \\
\hline SNT-TC-1A & Calificacion y certificacion de personal en ensayos no destructivos \\
\hline ASTM3 & $\begin{array}{l}\text { A } 6 \text { Requerimientos generales para laminas de acero rolado, moldes, } \\
\text { laminas apiladas y barras para uso estructural } \\
\text { A20 Requerimientos generales para laminas de acero en recipientes a } \\
\text { presion } \\
\text { A36 Acero estructural } \\
\text { A370 metodo de prueba estandar y definicion para prueba mecanica de } \\
\text { productos de acero } \\
\text { A992 Acero para formas estructurales para uso en estructuras en } \\
\text { estructuras de construcciones }\end{array}$ \\
\hline D1 & $\begin{array}{l}\text { 1. Codigo de soldadura Estructural - Acero } \\
\text { 1. Codigo de Soldadura Estructural - Acero inoxidable }\end{array}$ \\
\hline STD 2610 & $\begin{array}{l}\text { Diseño, construccion, operación, mantenimiento e inspeccion de } \\
\text { facilidades de tanques y terminales. }\end{array}$ \\
\hline
\end{tabular}

Fuente: autor

\section{Mantenimiento}

Se define como la acción eficaz para el mejoramiento de aspectos operativos importantes de un establecimiento como lo son la seguridad, funcionalidad, productividad, confort, imagen corporativa, salubridad e higiene. Provisionando la posibilidad de un deseado manejo de costos de operación. Sus objetivos son: 
- Bajar costos de mantenimiento.

- Mejorar recursos.

- Elevar la vida útil del tanque de mantenimiento.

La Misión del mantenimiento es garantizar el óptimo funcionamiento de los componentes industriales, a través de procedimientos para prevenir fallas, reparar daños para un mejor desempeño que se basa en los tres puntos siguientes:

- Disponibilidad de activos.

- Conservación de activos fijos.

- Administración de recursos.

- Clasificación del mantenimiento.

- El mantenimiento se clasifica en dos partes en el preventivo y correctivo.

Cuando se habla de mantenimiento correctivo, este se lo lleva a cabo cuando se produce un daño en las partes más sensibles del tanque o al alcanzar su tiempo de vida útil esperado del parte más vulnerable del tanque. El cambio de elementos deteriorados del depósito, como también el equipo de transporte y el sistema de seguridad en todas las operaciones de corte soldadura por el peligro que éstas llevan consigo, tomándose en consideración el diseño inicial o normas de seguridad.

Actúa solo cuando tiene un error en el sistema, si no hay falla será un mantenimiento nulo y este tiene que esperar que presente un error para tomar las medidas correctivas, como consecuencia puede llegar a ocasionar.

\section{Pasos para el mantenimiento correctivo}

Son cinco pasos que se numera a continuación:

- Reconocer fallas.

- Localizar.

- Diagnosticar.

- Reparar. 
- Revisar.

El mantenimiento preventivo es una inspección programada en funcionamiento como lo es su seguridad, limpieza, ajustes, reparaciones, etc., que se debe realizar con planificaciones realizadas. El fin es proveer los daños que pueden ser sujetos por diferentes acontecimientos como medio ambiente, tiempo de uso, etc. Este tiene el propósito de hacer el control preventivo de un tanque para poder evitar el deterioro anticipado del mismo para que no presente una avería. El potencial enemigo es la corrosión, es por eso que deben tomar ciertas medidas especiales para impedir que siga deteriorando el material del tanque. Con este mantenimiento se detectará fallos repetitivos, eliminará las paradas de producción, aumentará la vida útil y disminuirá costos de reparación.

De una manera resumida se puede observar en la Figura 1, las fases del mantenimiento específicamente para tanques.

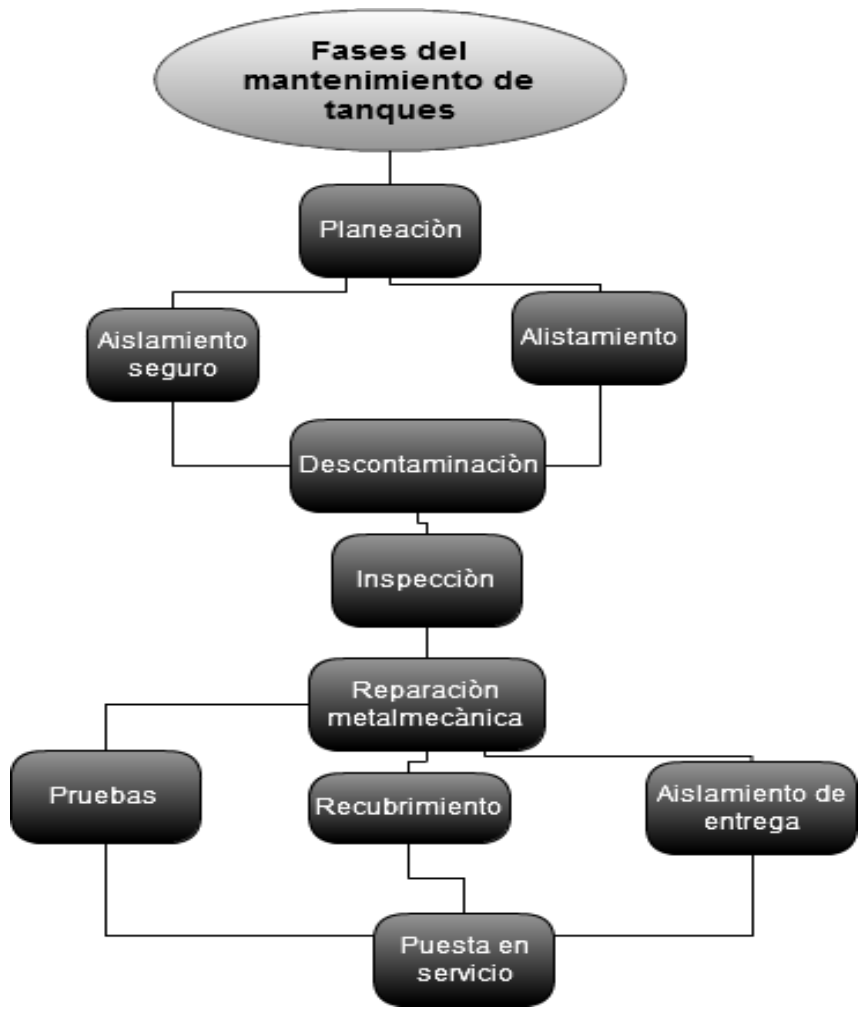

Figura 1. Fases del mantenimiento de los tanques

Fuente: Ecopetrol, 2011.

\section{Procedimiento al mantenimiento del tanque con fallas}


Los procedimientos que se siguen para el mantenimiento de tanques de domo geodésicos y para cualquier otro tanque son estándares de acuerdo a la política que sigue Petroecuador con la diferencia que se utilizan las normativas vigentes, en esta investigación se hablado en utilizar normativas API 653.

Para esta investigación se prevé un mantenimiento al tanque de domo geodésico, basándonos en los procedimientos realizados en Petroecuador.

Inspección interna: la inspección interna siguiendo los pasos de la normativa para los tanques de almacenamiento, se los debe realizar a través de intervalos de inspección e intervalos de inspección alternativa.

Intervalos de inspección: estos intervalos se los debe realizar a través de las ratas de corrosión que son las medidas que se las ha hecho previamente. También cuando los rangos de corrosión no se conocen y no hay experiencia para determinar el espesor mínimo de la lámina del fondo del tanque.

Intervalo de inspección externa alternativa: el propietario u operador puede establecer un intervalo de inspección interna usando el procedimiento de inspección basada en riesgos (RBI). Algunos de los factores que se deben considerarse en la evaluación RBI de un tanque incluyen lo siguiente:

- El material de construcción, incluyendo recubrimientos y pinturas, relativos a la temperatura del producto y las condiciones del ambiente.

- El estándar como-construido (As-Built).

- Los métodos usados para la determinación del espesor de la lámina del fondo y el cuerpo.

- La confianza y efectividad de los métodos de inspección y calidad de la información recolectada.

Evaluación de techos de tanques: Cuando se evalúan los techos de los tanques se debe tomar en cuenta la integridad y los soportes del techo. Los techos a ser evaluados son los de domo geodésico que están constituidos por aluminio con una estructura triangular diseñado como una bóveda auto portante el cual solo está conectado a un borde exterior a la pared del tanque. 


\section{Análisis y resultados}

Para lograr un excelente mantenimiento es necesario varios elementos que al fusionarlos armonicen y contribuyan a un funcionamiento continuo del tanque de almacenamiento. El manejo de los recursos está basado en la coordinación de acuerdo a los mantenimientos y en los principios básicos de la administración, dirección y el control.

Para optimizar recursos se realizó un banco de tareas, donde se encuentra la información necesaria del tanque para llevar a cabo grupos de tareas con diferentes frecuencias de tiempo de mantenimiento de tanques de almacenamiento, los mismos están constituido de la siguiente manera.

- Parte del tanque: es el componente del tanque de almacenamiento de techo de domo geodésico, en el cual se va a realizar la tarea.

- Tarea: está relacionado con la actividad a desempeñarse en el tanque.

- Frecuencia: indica el intervalo de tiempo dentro del cual debe llevarse a cabo cada una de las tareas.

- Personal: él o las personas que deben realizar la respectiva tarea de mantenimiento.

- Tiempo estimado: tiempo estimado de duración que requiere para llevar a cabo la tarea de mantenimiento correspondiente.

- Descripción: indica el procedimiento a seguir para llevar a cabo cada una de las tareas.

- Herramientas: indica los diferentes tipos de herramientas que se requieren para la realización de las tareas de mantenimiento.

- Materiales: elementos fungibles que se requieren para la realización de una determinada tarea de mantenimiento.

- Equipos: componentes que se requieren para la realización de una determinada tareade mantenimiento.

Los pasos a seguir para cada proceso del mantenimiento son cuándo debe realizarse cada una de las tareas planificadas, teniendo en cuenta las capacidades que tiene la empresa para almacenar y 
procesar el crudo, la cantidad de los derivados, el material y equipos a utilizar, y la mano de obra disponible. A continuación, se describen actividades:

1. Revisar y analizar los reportes de inspección anteriores para tener un punto de referencia para realizar la inspección.

2. Llenar la solicitud de permiso de trabajo para luego presentarlo y que firme la aceptación.

3. Realizar las tareas establecidas para esa fecha o día de trabajo.

4. Luego acudir a la oficina donde fue aceptada la solicitud de trabajo para cerrar el permiso de trabajo.

5. Recopilar todos los datos e imágenes y realizar el reporte de inspección otrabajos.

Tabla 2. Formato actividades para la inspección externa de rutina de tanques atmosféricos

\begin{tabular}{|c|c|c|c|c|c|}
\hline $\begin{array}{l}\text { EPETROECUADOR } \\
\text { ATP. UNDAD DENSPECCIONTECNCA } \\
\text { ATP SETRIA }\end{array}$ & RUTINA & IRA TANQU & ATMOSFÉRICOS. & & cuador \\
\hline Tanque: & Capacidad: & Producto: & Especific. Material : & Diámetro: & Altura: \\
\hline Y-T8023 & 14100 & GASOLINA & & & \\
\hline \multicolumn{3}{|c|}{ NOMBRE DEL REPRESENTANTE: } & \multicolumn{2}{|c|}{ FRECUENCIA : } & $\begin{array}{l}\text { Mensu } \\
\text { al }\end{array}$ \\
\hline \multicolumn{3}{|l|}{ LUGAR Y FECHA: } & \multicolumn{2}{|c|}{ TIEMPO ESTIMADO: } & $\begin{array}{l}2 \\
\text { horas. }\end{array}$ \\
\hline
\end{tabular}

DESCRIPCIÓN:

1. Revisar y analizar los reportes de inspección anteriores para tener un punto de referencia para realizar la inspección.

2. Llenar la solicitud de permiso de trabajo para luego presentarlo y que firme la aceptación.

3. Realizar las tareas establecidas para esa fecha o día de trabajo.

4. Luego acudir a la oficina donde fue aceptada la solicitud de trabajo para cerrar el permiso de trabajo.

5. Recopilar todos los datos e imágenes y realizar el reporte de inspección o trabajos.

\begin{tabular}{|l|l|l|l|l|l|}
\hline No. & Actividad & Cumple & $\begin{array}{l}\text { No } \\
\text { cumple }\end{array}$ & N/A & Observaciones \\
\hline 1. & ANILLO DE CONCRETO & & & & \\
\hline a. & Base de concreto sin roturas, grietas, fugas, muestras de & & \\
\hline
\end{tabular}

Pol. Con. (Edición núm. 19) Vol. 3, No 5, mayo 2018, pp. 128-149, ISSN: 2550 - 682X 


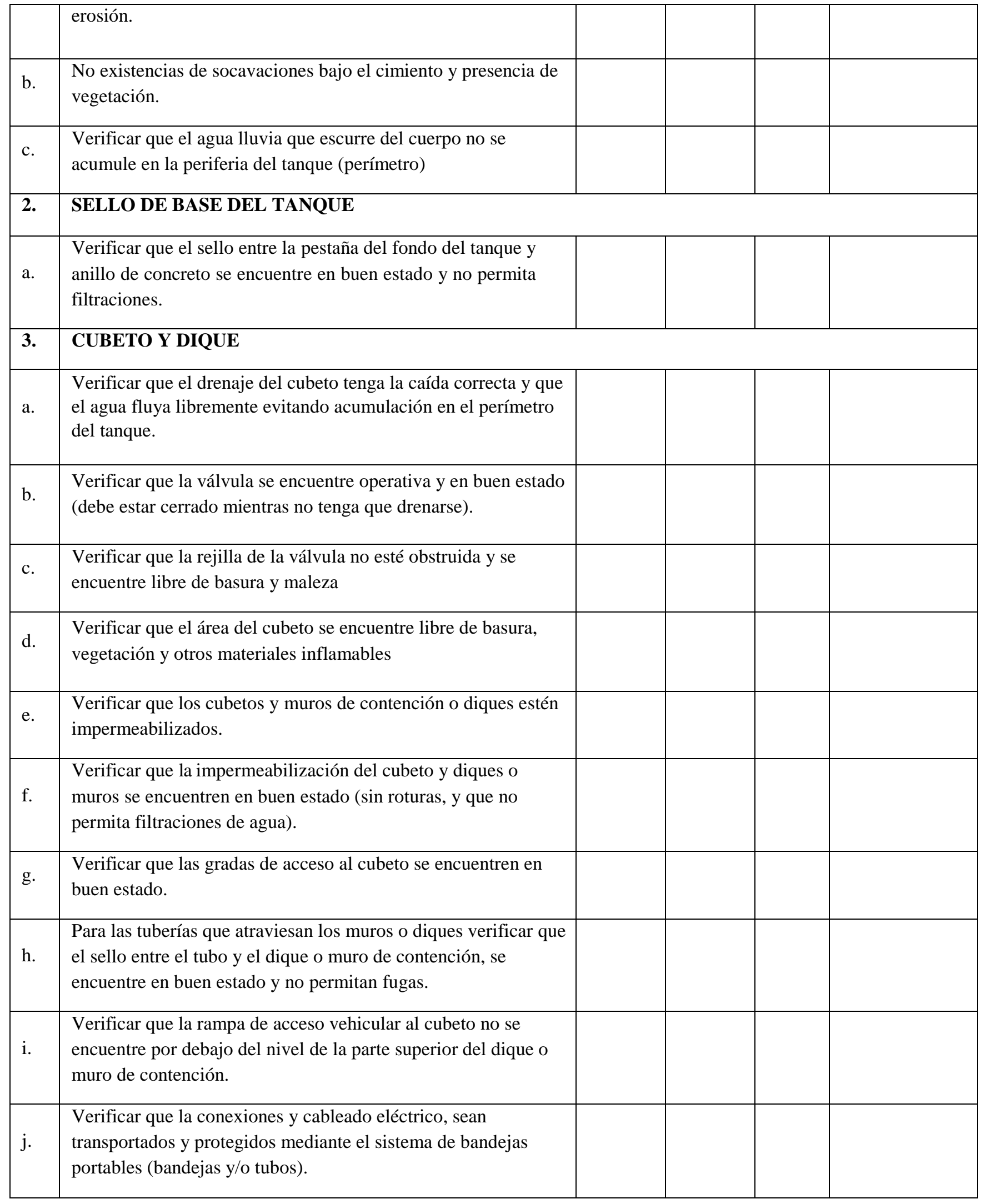





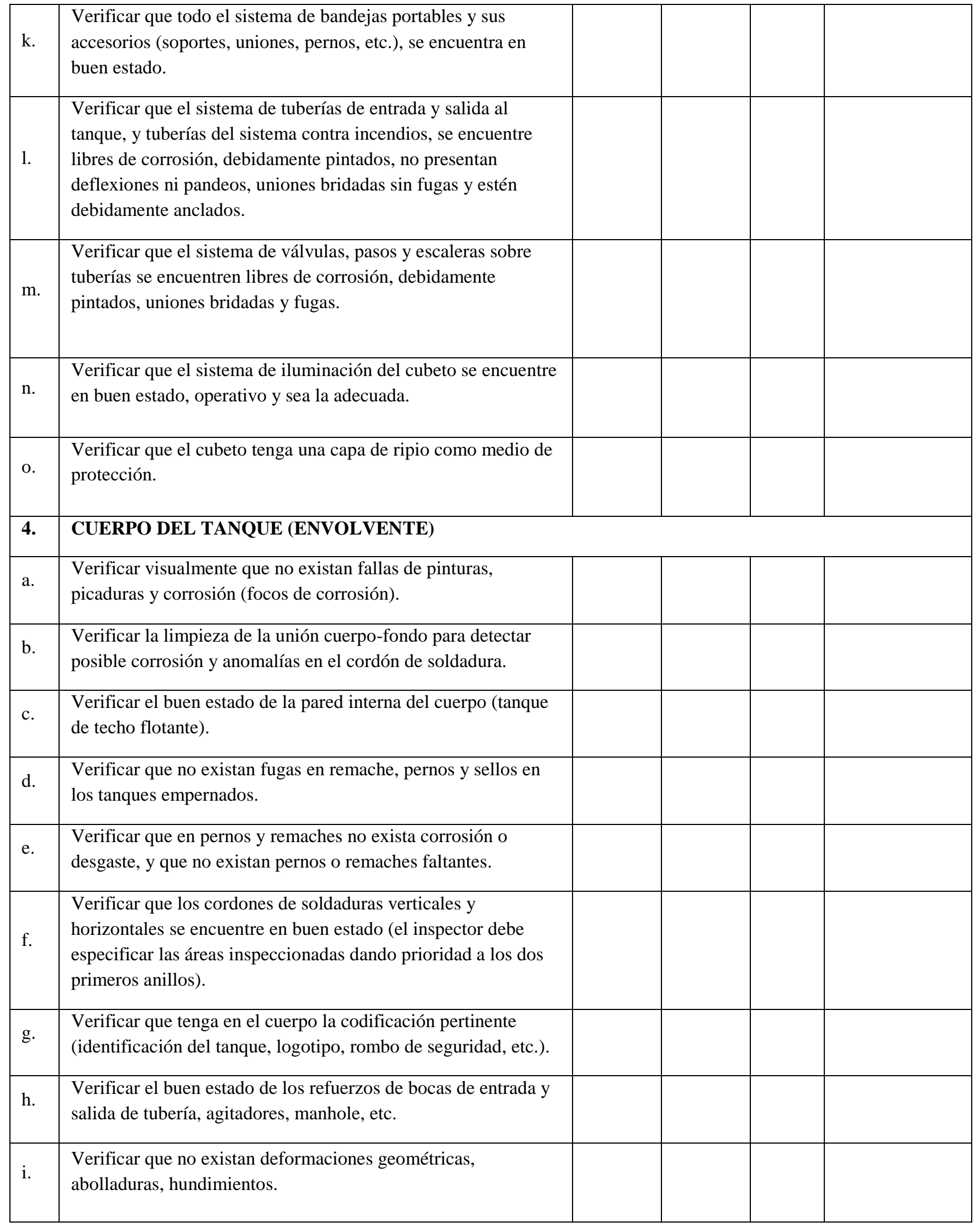




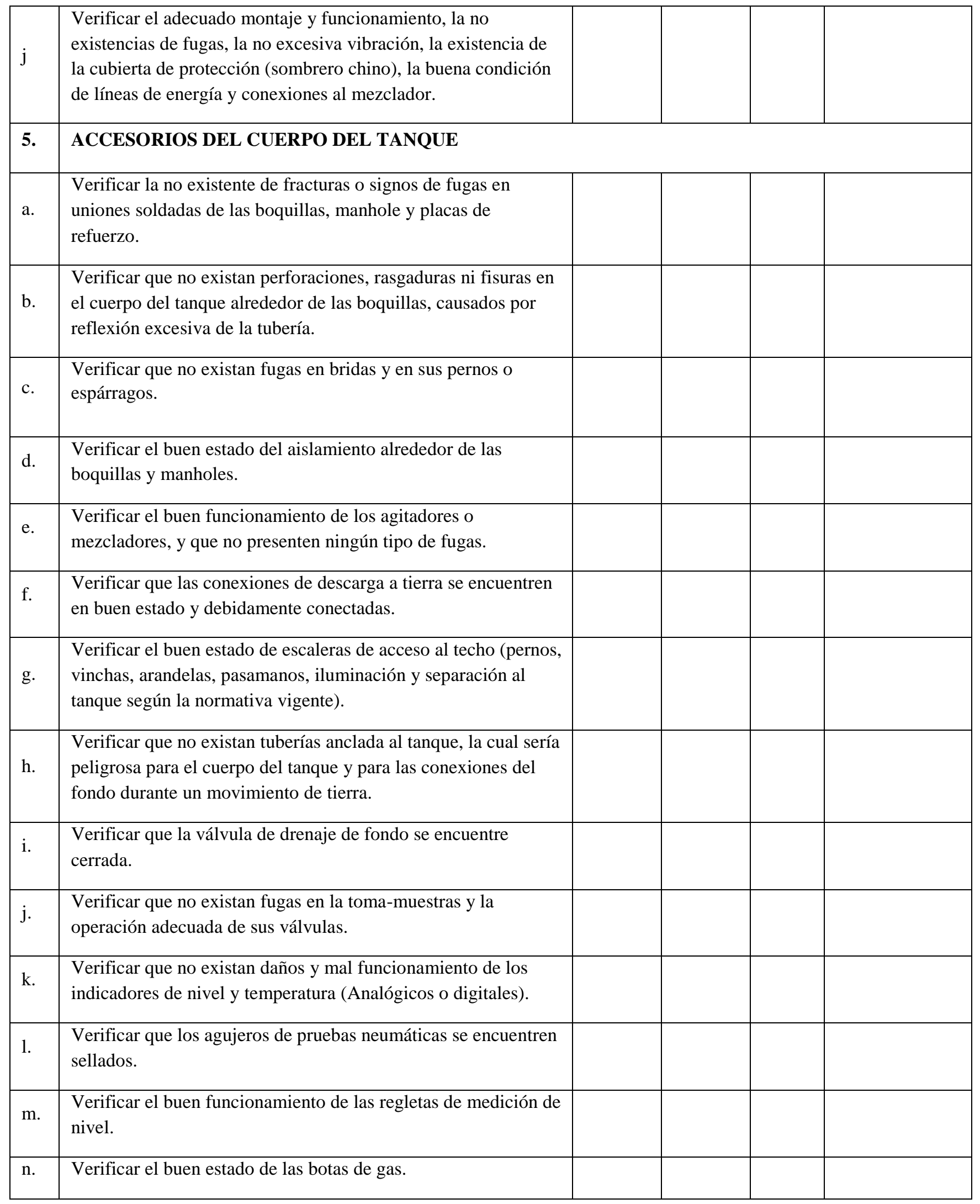




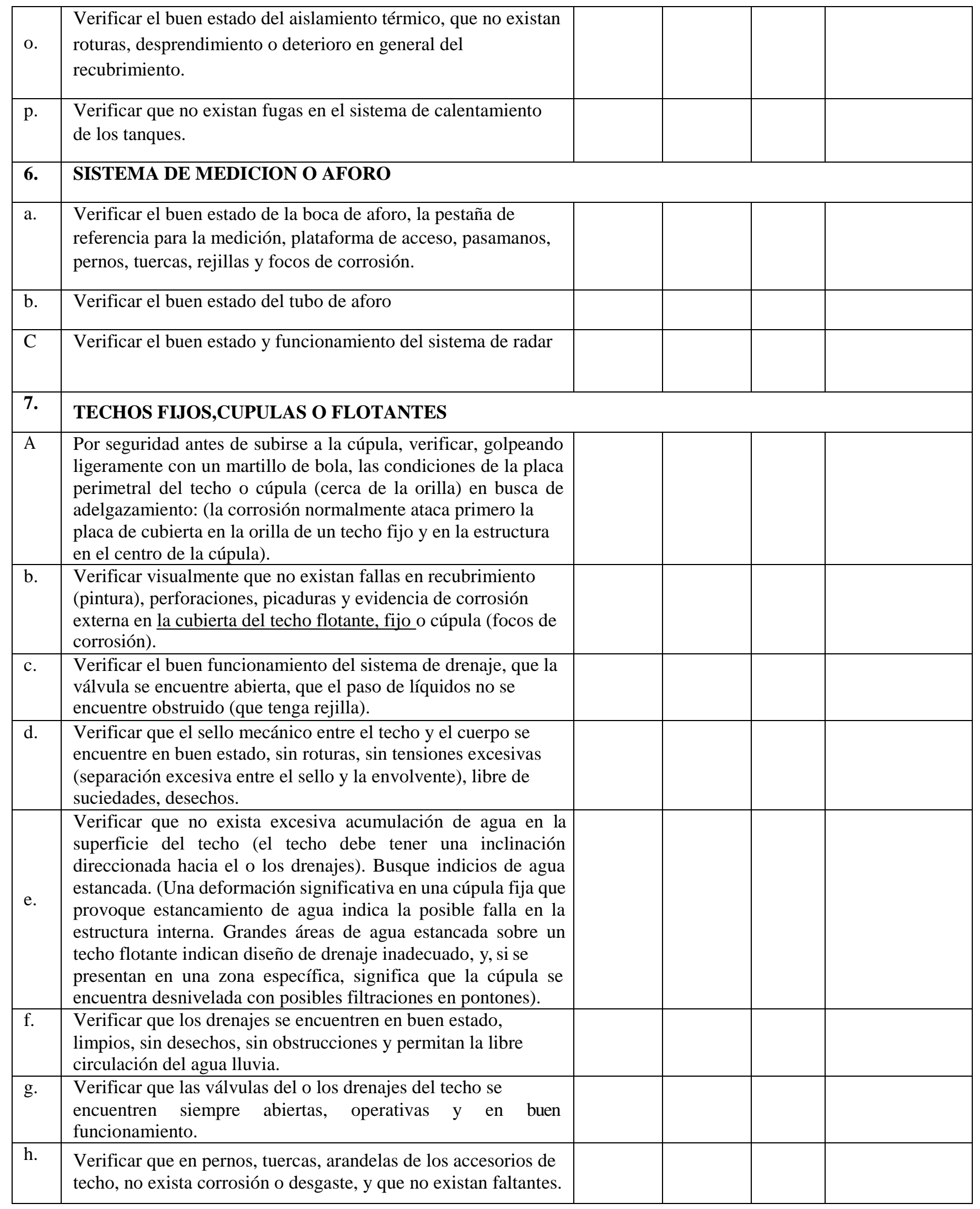




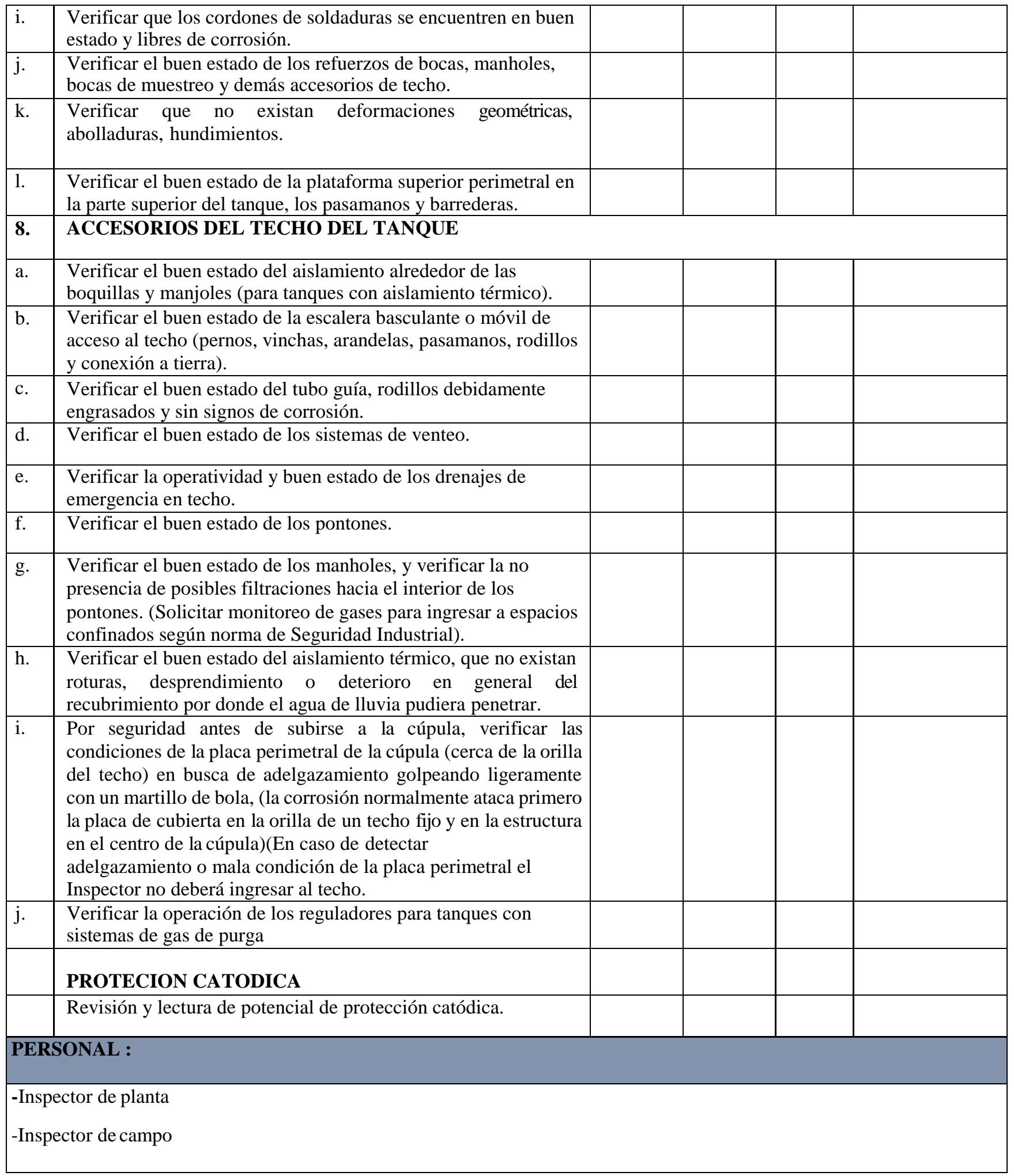

Fuente: autor 


\section{Análisis de costos del proyecto}

Los costos estimados para esta investigación, se corresponden a la implementación planificada de este plan de mantenimiento, estos han sido calculados para las actividades de mantenimiento con la frecuencia mensual, semestral, anual, cada 5 años, 10 años y 15 años. Es decir, contemplando la vida útil el equipo. A continuación, en las tablas posteriores se presentan estos cálculos mencionados.

Tabla 3. Costos indirectos del plan de mantenimiento

\begin{tabular}{|l|l|r|}
\hline Número & Denominación & V. Total \\
\hline 1 & Costos por ingeniería & 11149,59 \\
\hline 2 & Costos por herramientas utilizadas & 4164,04 \\
\hline 3 & Costo por imprevisto & 500 \\
\hline 4 & Utilidades & 0 \\
\hline & Total de costos indirectos & 15813,63 \\
\hline
\end{tabular}

Fuente: autor

Tabla 4. Costo de implementación del plan de mantenimiento

\begin{tabular}{|l|l|r|}
\hline Número & Denominación & V. Total \\
\hline 1 & Costo directo de equipos & 83280,83 \\
\hline 2 & Costo directo de materiales & 691,89 \\
\hline 3 & Costos indirectos & 15813,63 \\
\hline $\begin{array}{l}\text { Costo de implementación del plan de } \\
\text { mantenimiento }\end{array}$ & $99.786,35$ \\
\hline
\end{tabular}

Fuente: autor

Tabla 5. Costo total del plan de mantenimiento 


\begin{tabular}{|l|l|l|}
\hline \multicolumn{2}{|c|}{ Costo total del mantenimiento preventivo durante la vida útil } \\
\hline Número & Descripción & Valor \\
\hline 1 & Costo de mano de obra y materiales & 434774,61 \\
\hline 2 & Equipos y Herramientas & 83280,83 \\
\hline 3 & Costos indirectos & 15813,63 \\
\hline & Total del mantenimiento preventivo & 533869,08 \\
\hline
\end{tabular}

Fuente: autor

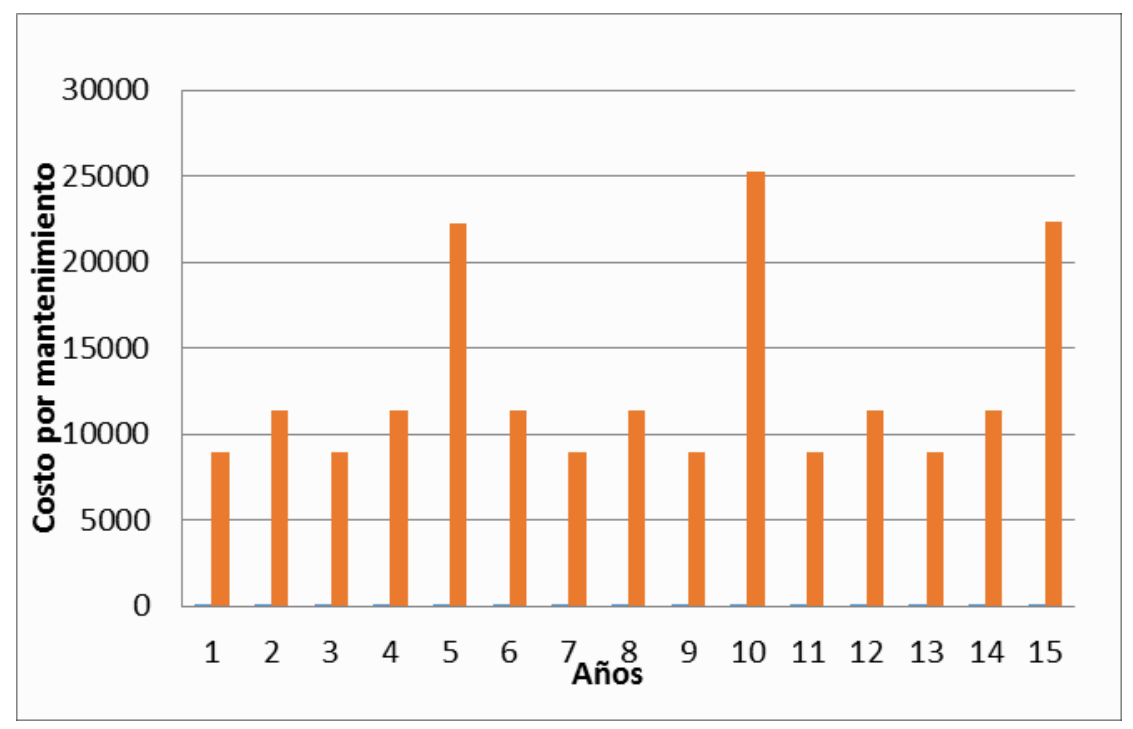

Figura 2. Relación de los años respecto al costo de mantenimiento

Fuente: autor

\section{Conclusiones}

Mediante este plan de mantenimiento basado en normas que nos guían a la reparación, mantenimiento e inspección de tanques de almacenamiento podemos realizar un diagnóstico preventivo para evitar o disminuir daños a largo y corto plazo, en base a sus necesidades y condiciones de operación, donde la gran mayoría se debe a una falta de planificación del mantenimiento.

Este plan esta acoplado a la demanda de las nuevas tecnologías de los tanques, con el fin de reducir el tiempo en el proceso de ejecución de mantenimiento y los costos de mano de obra de 
personal capacitado, produciendo así el ahorro de los recursos destinados para el mantenimiento, evitando paros de planta no deseados y perjudiciales para la economía de la empresa y del estado.

Brinda la facilidad de llevar a cabo un historial de las inspecciones de los fallos encontrados durante su vida útil donde podemos analizar detalladamente los puntos críticos del tanque donde más se producen desgaste, en base a las actividades respectivas para que de esa manera la integridad del tanque se encuentre en las mejores condiciones de servicio.

El listado de actividades desarrolladas permitirá preservar a los tanques instalados en continua operación, evitaran siniestros y el rápido desgaste del mismo donde estos trabajos se deben realizar por personal calificado por el riesgo que conlleva su inspección y trabajos de mantenimiento, las mismas que se debe realizar con periodicidad detalladas en las tablas.

Se deben atender las particularidades del tipo de producto almacenado, para garantizar la vida útil del tanque a través de un control y mantenimiento riguroso, sin embargo, la alteración de las características de un producto a otro diferente al de diseño, también varía el rango de aceleración de corrosión. Insertar el plan de mantenimiento de forma integral en la empresa gestionando la utilización de las tablas de actividades que contienen, frecuencia de tiempo, personal, herramientas, materiales y equipos, el mismo que proporciona la seguridad y calidad para el desarrollo y mejoramiento de sus tanques.

\section{Referencias Bibliográficas}

ASHRAE, (1990). Refrigeración, Sistemas y aplicaciones

Batista, J y Godoy L. (2011). Investigación de causas de explosiones en una planta de almacenamiento de combustible en Puerto Rico, SCIPEDIA, vol. 11, nº 2, pp. 110-122

Betún, C y Morillo, A (2010). Desarrollo de un plan de mantenimiento para tanques de almacenamiento de Petróleo Mantenimiento de PETROECUADOR para el terminal marítimo de Bilba, Quito: EPN

Campbell, J y Abdul (2000). Sistemas de mantenimiento: Planeación y control., México D.F.: Limusa 
Cuatrecasas Arbos, L (2000). Hacia la competitividad a través de la eficiencia de los equipos de producción, Barcelona: Gestión

Dounce Villanueva, E. (2000). Productividad en el Mantenimiento Industrial, México: Compañía Editorial Continental

ECOPETROL, (2015). «ECOPETROL,» [En línea]. Available: https://www.ecopetrol.com.co/wps/portal/es. [Último acceso: 312 2015].

ENERGY API, (2015). «API ORG,» API, [En línea]. Available: http://www.api.org/. [Último acceso: 312 2015]

García Garrido, S. (2003). Organización y gestión integral del mantenimiento, Madrid: Ediciones Diaz de Santos, 2003

Mayorga, M (2013). Inspección física y análisis estructural para determinar operatividad de un tanque cilíndrico vertical para almacenamiento de fuel oil de acuerdo a la norma API 653, Guayaquil: ESPOL

Murray, J (1997). Mantenimiento centrado en la confiabilidad, United Kingdom: Regent Street Naranjo M (2016). Plan de para tanques de Techo de domo geodesico, Riobamba: ESPOCH PETROECUADOR, (2000). «PETROECUADOR E.P.,». [En línea]. Available: https://www.eppetroecuador.ec/

R. Galván R, Reyes L, Guédez R y De Armas C. (2007). «Los Macroprocesos de la Industria Petrolera y sus consecuencias ambientales,» Universidad, Ciencia y Tecnología, vol. 11, nº 43, pp. $1-22$

Stoecker, W (1998). Industrial refrigeration handbook

Torres, L. (2005). Mantenimiento: su implementación y gestión, Córdoba: Universitas

Granda, V (2014). Diseño e implementación de un plan de mantenimiento para EP-

PETROECUADOR para la estación No. 1 Lago Agrio según la Norma API 653, Quito: ESPE

V. R. (2010). Apuntes de refrigeración, Riobamba 